Pure and Applied Mathematics Quarterly

Volume 2, Number 2

(Special Issue: In honor of

John H. Coates, Part 2 of 2$)$

497-518, 2006

\title{
Eigenvalues of Frobenius and Hodge Numbers
}

\author{
Mark Kisin and Gus Lehrer \\ To John Coates on his $60^{\text {th }}$ birthday
}

\section{INTRODUCTION}

We study the connection between Hodge purity of the cohomology of algebraic varieties over fields of different characteristics. Specifically, we study varieties over number fields, whose cohomology in some fixed degree $2 i$ consists entirely of Hodge classes, that is, whose Hodge cohomology in degree $2 i$ is entirely of type $(i, i)$. Among other things, we give sufficient conditions for varieties over number fields to satisfy this property in terms of their rational points over finite fields.

The proofs use arguments from $p$-adic Hodge theory, as well as the computation of points of a variety over finite fields via the Grothendieck-Lefschetz fixed point theorem.

Our results are applied to examples from the theory of reductive algebraic groups, including the fibres of the Grothendieck-Springer resolution, configuration spaces, the varieties of regular semisimple elements (elements in "general position") in Lie algebras and algebraic groups, and toric varieties.

The results are also applied to relate topological properties of the space $X(\mathbb{R})$ of real points of an algebraic variety to the set of its rational points over finite fields. A typical statement of this type is that for certain $X$, the Euler characteristic of $X(\mathbb{R})$ is obtained essentially by putting $q=-1$ in a polynomial which counts the number of points of $X\left(\mathbb{F}_{q}\right)$, and that this is equal to the dimension of the cohomology ring of $X$. When the connected components of $X(\mathbb{R})$ are known to be contractible, this result counts these components.

Received August 3, 2005.

M. Kisin is partially supported by NSF grant DMS-0400666 and a Sloan Research Fellowship. G. Lehrer is supported by an Australian Research Council Professorial Fellowship; he also thanks the Centre Bernoulli, EPFL Lausanne for hospitality during the preparation of this work. 
After the writing of this paper was completed, we learnt of the recent article of Edixhoven-van den Bogaart [EvdB], which gives a criterion under which the cohomology of a smooth proper stack is of Tate type, in terms of the points of the stack in finite fields. Although the emphasis of that paper is rather different, the basic idea, using $p$-adic Hodge theory, is similar.

It is a pleasure to thank the referee for a careful reading of the paper and many valuable suggestions.

\section{$\S 1 p$-ADIC Hodge THEORY}

(1.1) We begin with a general result about Galois representations. Suppose that $F$ is a number field, $S$ is a finite set of places of $F, \bar{F}$ is an algebraic closure of $F$, and that $F_{S} \subset \bar{F}$ is the maximal extension of $F$ which is unramified outside $S$. Let $G_{F, S}=\operatorname{Gal}\left(F_{S} / F\right)$.

If $v \notin S$ is a place of $F$, we denote by Frob $_{v} \in G_{F, S}$ the corresponding geometric Frobenius automorphism, which is well defined up to conjugation in $G_{F, S}$ [La, $\S 1.5]$. Set $q_{v}=|\kappa(v)|$, where $\kappa(v)$ denotes the residue field of $v$.

Let $p$ be a prime number, such that $S$ contains all primes of $F$ which divide $p$. Denote by $\mathbb{Q}_{p}(1)$, the one dimensional representation of $G_{F, S}$ over the field $\mathbb{Q}_{p}$ of $p$-adic numbers given by the cyclotomic character $\chi_{p}: G_{F, S} \rightarrow \mathbb{Z}_{p}^{\times}$, and for $i \in \mathbb{Z}$, set $\mathbb{Q}_{p}(i)=\mathbb{Q}_{p}(1)^{\otimes i}$.

For each prime $\mathfrak{p}$ of $F$ dividing $p$, we fix an algebraic closure $\bar{F}_{\mathfrak{p}}$ of the completion $F_{\mathfrak{p}}$ of $F$ at $\mathfrak{p}$. Fix an embedding $\bar{F} \hookrightarrow \bar{F}_{\mathfrak{p}}$, and denote the corresponding decomposition group by $G_{F_{\mathfrak{p}}}$; this data determines a homomorphism $G_{F_{\mathfrak{p}}} \rightarrow G_{F, S}$. In particular, any representation of $G_{F, S}$ may be restricted to $G_{F_{\mathrm{p}}}$. For any representation of $G_{F, S}\left(\right.$ resp. $\left.G_{F_{\mathfrak{p}}}\right)$ on a $\mathbb{Q}_{p}$-vector space $U$ we set $U(i):=U \otimes \mathbb{Q}_{p}(i)$.

We use the standard notation $B_{\mathrm{dR}}$ for the filtered ring defined by Fontaine [Fo1, $\S 1]$. This is a discretely valued field which contains $F_{\mathfrak{p}}$, and whose construction depends functorially on $\bar{F}_{\mathfrak{p}}$. Its residue field is canonically isomorphic to the $p$ adic completion of $\bar{F}_{\mathfrak{p}}$, which we shall denote by $\mathbb{C}_{p}$. In the next few paragraphs, we summarise some properties of $B_{\mathrm{dR}}$ which are relevant for our applications.

The field $B_{\mathrm{dR}}$ contains a uniformiser $t$ on which $G_{F_{\mathrm{p}}}$ acts via the cyclotomic character. That is, for $g \in G_{F_{\mathfrak{p}}}$ we have $g(t)=\chi_{p}(g) \cdot t$. The valuation on $B_{\mathrm{dR}}$ gives rise to a decreasing filtration $\mathrm{Fil}^{\bullet} B_{\mathrm{dR}}$, in which for $i \in \mathbb{Z}$, $\mathrm{Fil}^{i} B_{\mathrm{dR}}$ consists of the elements with valuation at least $i$. If we abuse notation by again writing $t$ for the image of $t$ in $\mathrm{Gr}^{1} B_{\mathrm{dR}}$, then we have

$$
\operatorname{Gr}^{\bullet} B_{\mathrm{dR}} \stackrel{\sim}{\longrightarrow} \oplus_{i \in \mathbb{Z}} \mathbb{C}_{p}(i) \stackrel{\sim}{\longrightarrow} \mathbb{C}_{p}\left[t, t^{-1}\right] .
$$


Now a result of Tate asserts that the subspace of $G_{F_{\mathfrak{p}}}$-invariant elements $\mathbb{C}_{p}(i)^{G_{F_{\mathfrak{p}}}}$ is equal to $F_{\mathfrak{p}}$ if $i=0$ and is $\{0\}$ otherwise. It follows by dévissage that

$$
B_{\mathrm{dR}}^{G_{F_{\mathfrak{p}}}}=F_{\mathfrak{p}}=\left(\mathrm{Gr}^{\bullet} B_{\mathrm{dR}}\right)^{G_{F_{\mathfrak{p}}}} .
$$

For any finite dimensional $\mathbb{Q}_{p}$-vector space $V$ with a continuous $G_{F_{\mathrm{p}}}$-action, we write $D_{\mathrm{dR}, \mathfrak{p}}(V)=\left(B_{\mathrm{dR}} \otimes_{\mathbb{Q}_{p}} V\right)^{G_{F_{\mathfrak{p}}}}$. This is a finite dimensional $F_{\mathfrak{p}}$-vector space with a decreasing filtration induced by that of $B_{\mathrm{dR}}$. For any such $V$, one has $\operatorname{dim}_{F_{\mathfrak{p}}} D_{\mathrm{dR}, \mathfrak{p}}(V) \leqslant \operatorname{dim}_{\mathbb{Q}_{\mathfrak{p}}} V$, and $V$ is said to be de Rham if $\operatorname{dim}_{F_{\mathfrak{p}}} D_{\mathrm{dR}, \mathfrak{p}}(V)=$ $\operatorname{dim}_{\mathbb{Q}_{p}} V$. Since the functor $D_{\mathrm{dR}}$ is evidently left exact, given a short exact sequence of finite dimensional $\mathbb{Q}_{p}$-representations of $G_{F_{\mathfrak{p}}}$

$$
0 \rightarrow V_{1} \rightarrow V_{2} \rightarrow V_{3} \rightarrow 0
$$

we obtain an exact sequence

$$
0 \rightarrow D_{\mathrm{dR}, \mathfrak{p}}\left(V_{1}\right) \rightarrow D_{\mathrm{dR}, \mathfrak{p}}\left(V_{2}\right) \rightarrow D_{\mathrm{dR}, \mathfrak{p}}\left(V_{3}\right) .
$$

Hence, by dimension, if $V_{2}$ is de Rham, then so are $V_{1}$ and $V_{3}$; however the converse is false. This implies that the category of de Rham representations is closed under the taking of subquotients, a fact which is crucial later.

Proposition (1.2). Let $V$ be a finite dimensional $\mathbb{Q}_{p}$-vector space, equipped with a continuous action of $G_{F, S}$. Assume that

(1) There exists an integer $i$ such that for all $v \notin S$, the eigenvalues of Frob $v$ acting on $V$ are of the form $\zeta q_{v}^{i}$ where $\zeta$ is a root of unity.

(2) For any prime $\mathfrak{p}$ of $F$ which divides $p$, the restriction $\left.V\right|_{G_{F_{\mathfrak{p}}}}$ (see above) is de Rham.

Then the action of $G_{F, S}$ on $V(i)$ factors through a finite quotient.

Proof. Replacing $V$ by $V(i)$, we may assume that $i=0$, i.e. that Frobenius elements have eigenvalues which are roots of unity. Now for any $v \notin S$ the eigenvalues of Frob $v$ acting on $V$ are roots of unity contained in an extension of $\mathbb{Q}_{p}$ of degree $e \leqslant d$, where $d=\operatorname{dim}_{\mathbb{Q}_{p}} V$. Since there are only finitely many such roots of unity, there are only finitely many possibilities for the characteristic polynomial of $\operatorname{Frob}_{v}$. Now the function which takes $g \in G_{F, S}$ to $\operatorname{det}(T I-g \mid V) \in \mathbb{Q}_{p}[T]$, is continuous and takes only finitely many values. To see this last assertion, observe that by Chebotarev's density theorem, there is a sequence of Frobenius elements of $G_{F, S}$ with limit $g$, and by what we have seen above, a subsequence of these all have the same characteristic polynomial, which therefore is the characteristic polynomial of $g$. It follows that the set $\left\{g \in G_{F, S} \mid \operatorname{det}(T I-g \mid V)=(T-1)^{d}\right\}$ is open in $G_{F, S}$, and hence contains an open subgroup $G \subset G_{F, S}$.

Now if we replace $F$ by a suitable finite extension, $G_{F, S}$ will correspondingly be replaced by the above open subgroup $G$, which has finite index in $G_{F, S}$. Making these replacements, we may assume that $G_{F, S}$ acts unipotently on $V$. 
The condition (2) implies that for $\mathfrak{p} \mid p, \operatorname{dim}_{F_{\mathfrak{p}}} D_{\mathrm{dR}, \mathfrak{p}}(V)=d$. Since $V$ is an extension of copies of the trivial representation, we must have $\operatorname{Fil}^{0} D_{\mathrm{dR}, \mathfrak{p}}(V)=$ $D_{\mathrm{dR}, \mathfrak{p}}(V)$ and $\mathrm{Fil}^{1} D_{\mathrm{dR}, \mathfrak{p}}(V)=\{0\}$. It follows by [Se, Cor. of Thm 11] that the inertia subgroup $I_{\mathfrak{p}} \subset G_{F_{\mathfrak{p}}}$ acts on $V$ through a finite quotient which is in fact trivial, since the only unipotent element of finite order in $G L(V)$ is the identity. Finally [KW, 2.1] now implies that $G_{F, S}$ acts on $V$ through a finite quotient. (This is a consequence of class field theory.)

Observe that the proposition implies that $\operatorname{Frob}_{v}$ acts semi-simply on $V$, given the assumptions of (1.2).

\section{$\S 2$ Cohomology}

(2.1) By a variety $X$ over $F$ we shall mean a reduced scheme of finite type over $F$. (This is more general than the usual definition, but will be convenient here). There are three cohomology theories arising from this context, and we shall recall the relationships among them. Fix a non-negative integer $j$.

First, $H_{\mathrm{dR}}^{j}(X)$ will denote the de Rham cohomology of $X$ in degree $j$. This is an $F$-vector space with a decreasing filtration, the Hodge filtration, which we will denote by $\mathbf{F}^{\bullet} H_{\mathrm{dR}}^{j}(X)$. Secondly, for any embedding $\sigma: F \hookrightarrow \mathbb{C}$, set $X_{\sigma}=$ $X \otimes_{F} \mathbb{C}$, and denote by $X_{\sigma}(\mathbb{C})$ the corresponding complex analytic space. Then $H^{j}\left(X_{\sigma}(\mathbb{C}), \mathbb{C}\right)$ denotes the Betti cohomology of this space. Finally, denote by $H^{j}\left(X_{\bar{F}}, \mathbb{Q}_{p}\right)$, or simply $H^{j}\left(X, \mathbb{Q}_{p}\right)$, the $p$-adic étale cohomology of $X_{\bar{F}}:=X \otimes_{F} \bar{F}$. This is a $\mathbb{Q}_{p}$-vector space equipped with a continuous action of $G_{F, S}$, where the set $S$ of primes of $F$ is taken to be sufficiently large that it contains the primes dividing $p$, and the action of $\operatorname{Gal}(\bar{F} / F)$ on $p$-adic étale ohomology of $X$ factors through $G_{F, S}$.

It is known (see [De 2, §8.2] or [Di, Appendix C]) that the Betti cohomology $H^{j}\left(X_{\sigma}(\mathbb{C}), \mathbb{C}\right)$ has two natural filtrations. The first is the Hodge filtration $\mathbf{F}^{\bullet}$, which arises from the above Hodge filtration on the de Rham cohomology $H_{\mathrm{dR}}^{j}(X)$ by extension of scalars to $\mathbb{C}$, and the de Rham isomorphism:

$$
H_{\mathrm{dR}}^{j}(X) \otimes_{F} \mathbb{C} \stackrel{\sim}{\longrightarrow} H^{j}\left(X_{\sigma}(\mathbb{C}), \mathbb{C}\right) \stackrel{\sim}{\longrightarrow} H^{j}\left(X_{\sigma}(\mathbb{C}), \mathbb{Z}\right) \otimes_{\mathbb{Z}} \mathbb{C} .
$$

The second filtration is the complex conjugate filtration $\overline{\mathbf{F}}^{\bullet}$ of $\mathbf{F}^{\bullet}$. It is the image of $\mathbf{F}^{\bullet}$ under the map $1 \otimes c$ on the final term in (2.1.1), where $c$ denotes complex conjugation on $\mathbb{C}$.

There are two further relationships among these cohomology theories. Given an embedding $\bar{\sigma}: \bar{F} \hookrightarrow \mathbb{C}$ extending $\sigma$ and an embedding $\iota: \mathbb{Q}_{p} \hookrightarrow \mathbb{C}$, we have a canonical isomorphism between $p$-adic étale and Betti cohomology 


$$
H^{j}\left(X, \mathbb{Q}_{p}\right) \otimes_{\mathbb{Q}_{p}} \mathbb{C} \stackrel{\sim}{\longrightarrow} H^{j}\left(X_{\sigma}(\mathbb{C}), \mathbb{C}\right)
$$

Thirdly, given an embedding $\bar{F} \hookrightarrow \bar{F}_{\mathfrak{p}}$, we have an isomorphism of $B_{\mathrm{dR}}$-vector spaces.

$$
H_{\mathrm{dR}}^{j}(X) \otimes_{F} B_{\mathrm{dR}} \stackrel{\sim}{\longrightarrow} H^{j}\left(X, \mathbb{Q}_{p}\right) \otimes_{\mathbb{Q}_{p}} B_{\mathrm{dR}} .
$$

The isomorphism (2.1.3) is a consequence of the $p$-adic comparison isomorphism which was conjectured by Fontaine and Jannsen [Fo2, §6.1.2], and established in various degrees of generality by Fontaine-Messing, Kato, Faltings and Tsuji. A version which is general enough for our purposes is derived in [Ki, 3.3], using the results of Faltings [Fa].

Both sides of (2.1.3) are (independently) filtered $F_{\mathfrak{p}}$-vector spaces. On the left this filtration is the tensor product filtration of the Hodge filtration on $H_{\mathrm{dR}}^{j}(X)$ and the filtration on $B_{\mathrm{dR}}$. On the right it is induced by the filtration on $B_{\mathrm{dR}}$, so that $H^{j}\left(X, \mathbb{Q}_{p}\right) \otimes 1$ is deemed to be concentrated in degree zero. Similarly, both sides of (2.1.3) are equipped with a canonical action of $G_{F_{\mathfrak{p}}}$. On the right this action is via the action of $G_{F_{\mathfrak{p}}}$ on the two factors in the product

Lemma (2.1.4). The isomorphism (2.1.3) respects both the filtrations just described, and the action of $G_{F_{\mathfrak{p}}}$ on both sides.

Proof. The statement that the isomorphism (2.1.3) respects the $G_{F_{\mathfrak{p}}}$ action may be found in $[\mathrm{Ki}]$. The fact that the isomorphism of (2.1.3) respects the filtrations follows from the definition of the isomorphism in [Ki], the definition of the Hodge filtration in [De 2, 8.3.4], and the compatibility with filtrations of the $p$-adic comparison isomorphism of [Fa], on which [Ki] is based.

Note that the filtration of the left side of (2.1.3) is related to the Hodge filtration, while that of the right side arises purely from the Galois action. Thus the isomorphism (2.1.3) is the source of the relationship between geometry and arithmetic which is the crux of this note.

Taking $G_{F_{\mathfrak{p}}}$-invariants of both sides of (2.1.3) and using the fact that $B_{\mathrm{dR}}^{G_{F_{\mathfrak{p}}}}=$ $F_{\mathfrak{p}}$, we find that

$$
D_{\mathrm{dR}, \mathfrak{p}}\left(H^{j}\left(X, \mathbb{Q}_{p}\right)\right)=\left(H^{j}\left(X, \mathbb{Q}_{p}\right) \otimes_{F} B_{\mathrm{dR}}\right)^{G_{F \mathfrak{p}}} \stackrel{\sim}{\longrightarrow} H_{\mathrm{dR}}^{j}(X) \otimes_{\mathbb{Q}_{p}} F_{\mathfrak{p}} .
$$

By (2.1.1) and (2.1.2), the $F_{\mathfrak{p}^{-}}$-dimension of the right hand side is equal to the $\mathbb{Q}_{p}$-dimension of $H^{j}\left(X, \mathbb{Q}_{p}\right)$. Hence we see that $H^{j}\left(X, \mathbb{Q}_{p}\right)$ is de Rham, and it follows from the paragraph immediately preceding (1.2) that any subquotient of $H^{j}\left(X, \mathbb{Q}_{p}\right)$ is also de Rham. 
As explained in [De 3], each of these three cohomology theories carries an increasing weight filtration, which will be denoted $W_{\bullet}$. The construction is completely analogous to the one of [De 2]. A priori this depends on a choice of a simplicial resolution $Y^{\bullet} \rightarrow X$ of $X$ by smooth $F$-schemes $Y^{\bullet}$, together with a suitable compactification $Y^{\bullet} \hookrightarrow \bar{Y}^{\bullet}$. However, we have the following result.

Corollary (2.1.5). The weight filtration on each of the three cohomology theories above is independent of the choice of simplicial resolution $Y^{\bullet} \rightarrow X$.

Proof. First, recall that if we fix such a resolution, then each of the isomorphisms (2.1.1), (2.1.2) and (2.1.3) respects the relevant weight filtrations, where for this statement, $B_{\mathrm{dR}}$ is deemed to be concentrated in degree zero. This is a formal consequence of (a) the functoriality of each cohomology theory, and (b) the compatibility of these isomorphisms with Gysin maps (for the inclusion of a smooth cycle into a smooth proper variety). In the case of (2.1.1) and (2.1.2) (b) is standard, while in the case of (2.1.3) it follows from [Fa].

But it is shown in $\left[\right.$ De 3] that the weight filtration on $H^{j}\left(X_{\sigma}(\mathbb{C}), \mathbb{C}\right)$ is independent of the choice of resolution. The corresponding assertions for $H^{j}\left(X, \mathbb{Q}_{p}\right)$ and $H_{\mathrm{dR}}^{j}(X)$ now follow.

The three filtrations $\mathbf{F}^{\bullet}, \overline{\mathbf{F}}^{\bullet}$ and $W \bullet$ on $H^{j}\left(X_{\sigma}(\mathbb{C}), \mathbb{C}\right)$ satisfy the following relations [De 2, 8.3.3] (cf. also [Di, Appendix C, pp. 241 et seq.]). For any integers $n, p, q$ we have

$$
\mathbf{F}^{p} H^{j}\left(X_{\sigma}(\mathbb{C}), \mathbb{C}\right) \cap \overline{\mathbf{F}}^{q} H^{j}\left(X_{\sigma}(\mathbb{C}), \mathbb{C}\right) \subset W_{p+q} H^{j}\left(X_{\sigma}(\mathbb{C}), \mathbb{C}\right)
$$

and

$$
\operatorname{Gr}_{n}^{W} H^{j}\left(X_{\sigma}(\mathbb{C}), \mathbb{C}\right)=\oplus_{p+q=n} \operatorname{Gr}_{\mathbf{F}}^{p} \operatorname{Gr}_{\mathbf{F}}^{q} H^{j}\left(X_{\sigma}(\mathbb{C}), \mathbb{C}\right)
$$

From now on we fix an embedding $\sigma: F \hookrightarrow \mathbb{C}$.

Theorem (2.2). Maintain the above notation. For any integer $j$, consider the finite dimensional continuous $\mathbb{Q}_{p} G_{F, S}$-module $H^{j}\left(X, \mathbb{Q}_{p}\right)$.

Let $I=\left\{i_{1}, \ldots i_{m}\right\}$ be a finite set of integers with $i_{1}<i_{2} \cdots<i_{m}$, and for each $i \in I$ let $r_{i}$ be a positive integer. Suppose that for any prime $v \notin S$ of $F$, and integer $i \in I$, Frob $_{v}$ acting on $H^{j}\left(X, \mathbb{Q}_{p}\right)$ has exactly $r_{i}$ eigenvalues of the form $\zeta q_{v}^{i}$, where $\zeta$ is a root of unity, and that these are all the eigenvalues of $\mathrm{Frob}_{v}$ on $H^{j}\left(X, \mathbb{Q}_{p}\right)$. Then given an embedding $\sigma: \bar{F} \hookrightarrow \mathbb{C}$,

(1) There is a finite extension $F^{\prime}$ of $F$ such that if $S^{\prime}$ denotes the set of primes of $F^{\prime}$ lying over $S$, then $H^{j}\left(X, \mathbb{Q}_{p}\right)$ has a $G_{F^{\prime}, S^{\prime}}$-stable filtration

$$
\{0\}=V_{0} \subset V_{1} \subset \cdots \subset V_{m}=H^{j}\left(X, \mathbb{Q}_{p}\right)
$$

by $\mathbb{Q}_{p}$-subspaces, such that $V_{k} / V_{k-1} \stackrel{\sim}{\longrightarrow} \mathbb{Q}_{p}\left(-i_{k}\right)^{r_{i_{k}}}$ for $k=1,2 \ldots m$. 
(2) $\operatorname{Gr}_{\mathbf{F}}^{p} \operatorname{Gr}_{\mathbf{F}}^{q} H^{j}\left(X_{\sigma}(\mathbb{C}), \mathbb{C}\right)$ has dimension $r_{i}$ if $p=q=i$, with $i \in I$, and is 0 otherwise.

Proof. By functoriality, the weight filtration $W_{\bullet}$ of $H^{j}\left(X, \mathbb{Q}_{p}\right)$ is stable under the action of $G_{F, S}$. Let $V_{0}=\{0\}$ and for $k=1,2, \ldots m$, set $V_{k}=W_{2 i_{k}} H^{j}\left(X, \mathbb{Q}_{p}\right)$. It follows from the compatibility of the weight filtration defined above with the one defined using eigenvalues of Frobenius [De 3], that $V_{k} / V_{k-1}$ has dimension $r_{i_{k}}$, and that the eigenvalues of Frob $v$ on this space are all of the form $\zeta q_{v}^{i_{k}}$ for some root of unity $\zeta$.

As explained in $(2.1), H^{j}\left(X, \mathbb{Q}_{p}\right)$ is de Rham, whence so is $V_{k} / V_{k-1}$ by the remarks at the end of (1.1). Applying (1.2) to $V_{k} / V_{k-1}$ we see that there is a subgroup $G$ of of finite index in $G_{F, S}$ such that $G$ acts trivially on the modules $V_{k} / V_{k-1}\left(i_{k}\right)=\mathrm{Gr}_{2 i_{k}}^{W} H^{j}\left(X, \mathbb{Q}_{p}\right)\left(i_{k}\right)$. The statement (1) follows.

Now fix a prime $\mathfrak{p}$ of $F$ dividing $p$, and an embedding $\bar{F} \hookrightarrow \bar{F}_{\mathfrak{p}}$ as in (1.1). Since the truth of (2) is not affected if we replace $F$ by $F^{\prime}$, and $X$ by its base change to $F^{\prime}$, we may assume that $G=G_{F, S}$. From (2.1.3) we have for any integer $m$,

$$
\mathrm{Gr}_{m}^{W} H_{\mathrm{dR}}^{j}(X) \otimes_{F} B_{\mathrm{dR}} \cong \mathrm{Gr}_{m}^{W} H^{j}\left(X, \mathbb{Q}_{p}\right) \otimes_{\mathbb{Q}_{p}} B_{\mathrm{dR}}
$$

and this isomorphism is compatible with filtrations by (2.1.4). For any integer $n$, we may take the degree $n$ graded component of both sides, obtaining

$$
\oplus_{\ell} \operatorname{Gr}_{\mathbf{F}}^{\ell} \operatorname{Gr}_{m}^{W} H_{\mathrm{dR}}^{j}(X) \otimes_{F} \mathbb{C}_{p}(n-\ell) \cong \operatorname{Gr}_{m}^{W} H^{j}\left(X, \mathbb{Q}_{p}\right) \otimes_{\mathbb{Q}_{p}} \mathbb{C}_{p}(n) .
$$

Take $G_{F_{\mathfrak{p}}}$-fixed points in the last equation. Now $G_{F_{\mathfrak{p}}}$ acts trivially on $H_{\mathrm{dR}}^{j}(X)$, and since $\mathbb{C}_{p}(r)^{G_{F_{\mathfrak{p}}}}=F_{\mathfrak{p}}$ if $r=0$, and is zero otherwise, the left hand side becomes $\operatorname{Gr}_{\mathbf{F}}^{n} \mathrm{Gr}_{m}^{W} H_{\mathrm{dR}}^{j}(X) \otimes_{F} F_{\mathfrak{p}}$. To compute the $G_{F_{\mathfrak{p}}}$-invariants of the right hand side, observe that $\operatorname{Gr}_{m}^{W} H^{j}\left(X, \mathbb{Q}_{p}\right)=0$ unless $m=2 i_{k}$ for some $i_{k} \in I$. Moreover when this condition is satisfied, $\operatorname{Gr}_{m}^{W} H^{j}\left(X, \mathbb{Q}_{p}\right)$ is isomorphic to a direct sum of copies of $\mathbb{Q}_{p}\left(-i_{k}\right)$ as a $G_{F_{\mathfrak{p}}}$-representations. Hence the $G_{F_{\mathfrak{p}}}$-invariant part of the right side is 0 unless $(m, n)=\left(2 i_{k}, i_{k}\right)$ for some $i_{k} \in I$, and is equal to $\operatorname{Gr}_{2 i_{k}}^{W} H^{j}\left(X, \mathbb{Q}_{p}\right) \otimes_{\mathbb{Q}_{p}} F_{\mathfrak{p}}$ for $(m, n)=\left(2 i_{k}, i_{k}\right)$. It follows that $\operatorname{Gr}_{\mathbf{F}}^{n} \operatorname{Gr}_{m}^{W} H_{\mathrm{dR}}^{j}(X)=0$ unless $(m, n)=\left(2 i_{k}, i_{k}\right)$ for some $i_{k} \in I$, and is $\operatorname{Gr}_{2 i_{k}}^{W} H_{\mathrm{dR}}^{j}(X)$ if the condition is satisfied.

Hence using (2.1.1), we see that $\operatorname{Gr}_{2 i}^{W} H^{j}\left(X_{\sigma}(\mathbb{C}), \mathbb{C}\right)=\{0\}$ unless $i \in I$, and that for $k=1, \ldots m$, in the decomposition

$$
\operatorname{Gr}_{2 i_{k}}^{W} H^{j}\left(X_{\sigma}(\mathbb{C}), \mathbb{C}\right)=\oplus_{p+q=2 i_{k}} \operatorname{Gr}_{F}^{p} \operatorname{Gr}_{\bar{F}}^{q} H^{j}\left(X_{\sigma}(\mathbb{C}), \mathbb{C}\right),
$$

given by (2.1.6), the only non-zero term is the one where $p=i_{k}$, and so also $q=i_{k}$. This proves (2). 
Corollary (2.3). Let $X$ be a smooth projective variety over a number field $F$. Maintaining the above notation, the following are equivalent.

(1) For almost all places $v$ of $F$, the eigenvalues of $\operatorname{Frob}_{v}$ on $H^{2 j}\left(X, \mathbb{Q}_{p}\right)$ are of the form $\zeta q_{v}^{j}$, for $j=0,1,2, \ldots$, where $\zeta$ is a root of unity.

(2) For all $j, \mathrm{Gr}_{F}^{j} \operatorname{Gr}_{\bar{F}}^{j} H^{2 j}\left(X_{\sigma}(\mathbb{C}), \mathbb{C}\right)=H^{2 j}\left(X_{\sigma}(\mathbb{C}), \mathbb{C}\right)$.

Proof. The implication (1) $\Longrightarrow(2)$ is clear from (2.2). When $X$ is smooth and projective the converse is precisely the content of [KW, 1.3].

Remark (2.4). One might ask about a converse for $(2.2)(2)$ for general $X$. The proof of $[\mathrm{KW}]$ does apply, provided we make an assumption on the compatibility of the traces of Frobenius on various cohomology theories. Specifically, we need to assume that for $v \notin S$ the trace of $\operatorname{Frob}_{v}$ on $H^{j}\left(X, \mathbb{Q}_{p}\right)$ is independent of $p$ when $v \nmid p$, and is equal to the trace of Frobenius on the crystalline cohomology $H_{\text {cris }}^{j}\left(X_{F_{v}}\right)$ of $X_{F_{v}}=X \otimes_{F} F_{v}$ (where $F_{v}$ denotes the completion of $F$ at $v$.) This then implies the analogous compatibilities between the trace of Frobenius on the corresponding weight graded pieces of each of these cohomology theories.

Since $X_{F_{v}}$ may not be smooth or projective in general, there is more than one way to define the crystalline cohomology of $X_{F_{v}}$. (Of course different definitions conjecturally give rise to the same result). The most convenient definition (for example in order to be able to use a $p$-adic comparison isomorphism, and to compare with de Rham cohomology) is to take the crystalline cohomology of a simplicial resolution of $X_{F_{v}}$, in the style of [De 2].

As usual, the compatibility amongst traces of Frobenius does hold on the level of Euler characteristics, so it would suffice to assume that the same Frobenius eigenvalue cannot occur in the cohomology of $X$ in different degrees.

Remark (2.5). Each of the three cohomology theories $H^{*}$ with which we are concerned has a corresponding theory $H_{c}^{*}$ with compact supports. These are equipped with exactly the same structures (Hodge filtrations, Galois actions, weight filtrations) as their non-compact counterparts. Moreover the relations among these cohomology theories are completely analogous. Let us only remark that the existence of the mixed Hodge structure on $H_{c}^{*}$ (that is the Hodge, conjugate and weight filtrations satisfying (2.1.6)) follows from the theory of [De 2], since $H_{c}^{*}(X)$ can be expressed the usual cohomology of a simplicial scheme (cf. $[\mathrm{Ki}]$ ).

In particular the statement of Proposition (2.2), as well as its proof, go over verbatim with the cohomology $H^{j}$ replaced by cohomology with compact supports $H_{c}^{j}$.

We remark that when $X$ is smooth of dimension $d$, then by Poincaré duality, the condition in $(2.2)$ that the eigenvalues of Frobenius on $H^{j}\left(X, \mathbb{Q}_{p}\right)$ are of the 
form $\zeta q_{v}^{i}$ is equivalent to the condition that the eigenvalues on $H_{c}^{2 d-j}\left(X, \mathbb{Q}_{p}\right)$ are of the form $\zeta q_{v}^{d-i}$, which implies that $\operatorname{Gr}_{\mathbf{F}}^{d-p} \operatorname{Gr}_{\overline{\mathbf{F}}}^{d-q} H_{c}^{2 d-j}\left(X_{\sigma}(\mathbb{C}), \mathbb{C}\right)=\{0\}$ unless $p=q=i$ for some $i \in I$.

We finish this section with a definition.

Definition (2.6). Let $X$ be a variety over the number field $F$. Say that $X$ is mixed Tate if $H_{c}^{j}\left(X, \mathbb{Q}_{p}\right)$ satisfies the conditions of (2.2) (or (2.3)) for each $j$.

\section{$\S 3$ Mixed Tate varieties and points over finite fields}

In this section we give sufficient conditions for a variety $X$ to be mixed Tate, and exploit the conditions to give some examples.

We begin with the following simple observation.

Lemma (3.1). Suppose $X$ and $Y$ are mixed Tate varieties over a number field F. Then

(1) Suppose $W$ is a finite group acting on $X$ in such a way that the quotient variety $X / / W$ exists, (e.g. $X$ quasi-projective suffices). Then $X / / W$ is mixed Tate.

(2) Let $C$ be a closed subscheme of $X$ and $U=X \backslash C$. If any two of $X, C$ and $U$ are mixed Tate, so is the third.

(3) (cf. Eulerian collections $[D L, \S 2])$ Suppose $X$ is the union of a finite collection of closed subschemes $X_{\lambda}$ where $\lambda \in \Lambda$, which is closed under the taking of intersections. For each $\lambda \in \Lambda$, let $X_{\lambda}^{0}$ be the difference between $X_{\lambda}$ and the union of those $X_{\mu}$ such that $X_{\mu} \subsetneq X_{\lambda}$. Then all $X_{\lambda}$ are mixed Tate if and only if all $X_{\lambda}^{0}$ are mixed Tate.

(4) The product variety $X \times Y$ is mixed Tate.

Proof. The first statement follows by the transfer theorem, which asserts that for all $j$,

$$
H_{c}^{j}\left(X / / W, \mathbb{Q}_{p}\right) \stackrel{\sim}{\longrightarrow} H_{c}^{j}\left(X, \mathbb{Q}_{p}\right)^{W} \hookrightarrow H_{c}^{j}\left(X, \mathbb{Q}_{p}\right) .
$$

This isomorphism respects the action of the Galois group.

The second assertion follows easily from the excision sequence for cohomology with compact support. For the third claim, partially order $\Lambda$ by the inclusions among the $X_{\lambda}$. The statement (2) is the case of (3) where $|\Lambda|=1$. The statement is now proved by induction on $\lambda \in \Lambda$. One shows that for each $\lambda \in \Lambda, X_{\mu}$ is mixed Tate for each $\mu \leqslant \lambda$ if and only if $X_{\mu}^{0}$ is mixed Tate for each $\mu \leqslant \lambda$. For the induction step one again uses the excision sequence for cohomology with compact support.

The final claim follows from the Künneth formula for cohomology with compact support [SGA 4, XVII, 5.4.3] which applies because $X$ and $Y$ are compactifiable 
over $F$ by Nagata's theorem. The formula shows that the eigenvalues of Frob on $H_{c}^{j}\left(X \times Y, \mathbb{Q}_{p}\right)$ are products of the eigenvalues of $\mathrm{Frob}_{v}$ on $H_{c}^{k}\left(X, \mathbb{Q}_{p}\right)$ and on $H_{c}^{\ell}\left(Y, \mathbb{Q}_{p}\right)$, for $k+\ell=j$.

As an example of (3.1)(3) we have

Proposition (3.1.1). Let $V$ be a vector space over $F$, and let $V_{1}, \cdots, V_{r}$ be any collection of affine subspaces of $V$. Then the complement $V^{0}$ of the union of the $V_{i}$ is mixed Tate.

Proof. Clearly we may replace the collection $V_{i}$ by the set of all intersections of the $V_{i}$, and we arrive at the situation of $(3.1)(3)$. Since any affine space is mixed Tate, it follows that all the differences as defined in $(3.1)(3)$ are also mixed Tate; in particular, this is true of $V^{0}$.

Remark (3.1.2). A similar argument may be applied to show that the complement of a union of subtori in an algebraic torus is mixed Tate. Here we use the term torus to indicate a diagonalisable group, which need not necessarily be connected. The last two examples open possibilities for a combinatorial study of the precise weights which occur in the cohomology of the spaces concerned (see, e.g. [DL, Prop. 3.4]).

(3.2) In the following we will consider a variety $X$ over a number field $F$, and its points in finite fields. To make this precise, let $\mathcal{O}_{F}$ be the ring of integers of $F$, and let $\tilde{X}$ be a finite type $\mathcal{O}_{F}$-scheme with $\tilde{X} \otimes_{\mathcal{O}_{F}} F=X$. If $v$ is a prime of $\mathcal{O}_{F}$ with residue field $\kappa(v)$ and $\mathbb{F}_{q}$ is a finite extension of $\kappa(v)$, then we will write $X\left(\mathbb{F}_{q}\right)$ for $\tilde{X}\left(\mathbb{F}_{q}\right)$. This involves two abuses of notation. First $X\left(\mathbb{F}_{q}\right)$ depends on the place $v$ and not just on $\mathbb{F}_{q}$. Secondly $X\left(\mathbb{F}_{q}\right)$ depends on the choice of $\tilde{X}$. However two different choices of $\tilde{X}$ have the same $\mathbb{F}_{q}$-points for almost all choices of $v$. Thus the second abuse will be harmless provided we make statements for almost all primes $v$ of $F$.

Proposition (3.3). Suppose $X$ is a variety over a number field $F$ as in $\S 2$ and assume that for almost all primes $v$ of $F$, there is a power $q=q_{v}^{N_{v}}$ and a polynomial $P(t) \in \mathbb{Q}[t]$ such that for $m \geq 1$,

$$
\left|X\left(\mathbb{F}_{q^{m}}\right)\right|=P\left(q^{m}\right) .
$$

Then

(1) If $P(1)=\operatorname{dim} H_{c}^{*}\left(X, \mathbb{Q}_{p}\right)\left(:=\oplus_{j} H_{c}^{j}\left(X, \mathbb{Q}_{p}\right)\right)$ then $X$ is mixed Tate. Moreover $H_{c}^{j}\left(X, \mathbb{Q}_{p}\right)=0$ for $j$ odd.

(2) If $P(-1)=\varepsilon(X) \operatorname{dim} H_{c}^{*}\left(X, \mathbb{Q}_{p}\right)$, where $\varepsilon(X)= \pm 1$, then again $X$ is mixed Tate, and for each $j$, the Hodge filtration on $H_{c}^{j}\left(X_{\sigma}(\mathbb{C}), \mathbb{C}\right)$ is concentrated in degrees $i_{j}$ such that $(-1)^{i_{j}}=\varepsilon(X)(-1)^{j}$. 
Proof. The eigenvalues of $\mathcal{F}=\operatorname{Frob}_{v}^{N_{v}}$ are Weil numbers, i.e. are all of the form $\zeta q^{\frac{w}{2}}$, where $w$ is an integer, and $\zeta$ has absolute value 1 for any embedding $\mathbb{Q}_{p} \hookrightarrow \mathbb{C}$. Take a fixed embedding of $\mathbb{Q}_{p}$ in $\mathbb{C}$, and write

$$
\left\{\zeta_{i} q^{\frac{w_{i}}{2}} \mid 1 \leqslant i \leqslant d=\operatorname{dim} H_{c}^{*}\left(X, \mathbb{Q}_{p}\right)\right\}
$$

for the multi-set of eigenvalues of $\mathcal{F}$ on $H_{c}^{*}\left(X, \mathbb{Q}_{p}\right)$, regarded as elements of $\mathbb{C}$.

By Grothendieck's fixed point formula,

$$
\left|X\left(\mathbb{F}_{q^{m}}\right)\right|=\sum_{j}(-1)^{j} \operatorname{Trace}\left(\mathcal{F}^{m}, H_{c}^{j}\left(X, \mathbb{Q}_{p}\right)\right) .
$$

For $i=1, \cdots, d$ write $d(i)$ for the cohomology degree in which the eigenvalue $\zeta_{i} q^{\frac{w_{i}}{2}}$ occurs (note that we do not assume here that a given eigenvalue occurs in only one cohomology degree). Then

$$
P\left(q^{m}\right)=\sum_{i=1}^{d}(-1)^{d(i)}\left(\zeta_{i} q^{\frac{w_{i}}{2}}\right)^{m} .
$$

Since this holds for any $m \geq 1$, we see that the coefficient of $q^{\frac{w_{i}}{2} m}$ on the right hand side is 0 if $w_{i}$ is odd. That is, if $w$ is odd, then $\sum_{w_{i}=w}(-1)^{d(i)} \zeta_{i}^{m}=0$. The assumption in (1) now implies that

$$
d=P(1)=\sum_{i: 2 \mid w_{i}}(-1)^{d(i)} \zeta_{i}^{m} \leqslant \sum_{i=1}^{d}\left|\zeta_{i}\right|=d .
$$

It follows that $2 \mid w_{i}$ for each $i$, and $\zeta_{i}^{m}=(-1)^{d(i)}$. Taking $m=1,2$, we see that $d(i)$ is even and that $\zeta_{i}=1$.

Finally, it follows that the eigenvalues of $\operatorname{Frob}_{v}$ have the form $\zeta_{i}^{\prime} q_{v}^{\frac{w_{i}}{2}}$ where $\left(\zeta_{i}^{\prime}\right)^{N_{v}}=\zeta_{i}$. In particular, the $\zeta_{i}^{\prime}$ are roots of unity, and $X$ is mixed Tate. This completes the proof of (1).

A similar argument shows that under the assumption of (2), $w_{i}$ is even, $\zeta_{i}=1$, and $(-1)^{d(i)+\frac{w_{i}}{2}}=\varepsilon(X)$, for each $i$. As above, this shows that $X$ is mixed Tate, and further that for each $i,(-1)^{d(i)}=\varepsilon(X)(-1)^{\frac{w_{i}}{2}}$, as asserted.

We remark that although a priori the polynomial $P(t)$ may depend on $v$, the theorem together with (2.2) implies that it is independent of $v$. The following converse of (3.3) follows immediately from (2.2).

Proposition (3.4). Let $X$ be a mixed Tate variety over the number field $F$. Then there exists a polynomial $P(t) \in \mathbb{Q}[t]$ such that for almost all primes $v$ of $F$, there is a power $q=q_{v}^{N_{v}}$ such that $\left|X\left(\mathbb{F}_{q^{m}}\right)\right|=P\left(q^{m}\right)$ for $m \geq 1$.

(1) If the cohomology of $X$ is concentrated in even degrees, then $X$ satisfies the conditions of (3.3)(1). 
(2) If there is an integer $\varepsilon(X)= \pm 1$ such that the Hodge filtration degrees $i_{j}$ are related to the cohomology degrees $j$ by $(-1)^{i_{j}}=\varepsilon(X)(-1)^{j}$, then $X$ satisfies the conditions of (3.3)(2).

Examples. We give some examples from the theory of reductive algebraic groups. Let $G$ be a connected reductive group scheme defined over $F$, where $F$ is a number field as above. Let $T$ be a maximal torus, and let $\mathcal{B}^{G}=\mathcal{B}$ be the associated flag variety, whose points in an extension $K$ of $F$ are the Borel subgroups of $G(K)$.

(3.5) The cohomology of $G / T(K)$, which clearly is a module for the Weyl group $W:=N_{G}(T) / T$, is well-known by a result of Borel to be graded isomorphic to the coinvariant algebra of the Weyl group $W$. Thus $\operatorname{dim} H^{*}(G / T)=|W|$. But it is well known (see, e.g., [L]) that $\left|G / T\left(\overline{\mathbb{F}}_{q}\right)^{\mathcal{F}}\right|=q^{\left|\Phi^{+}\right|} \prod_{i} \frac{1-q^{d_{i}}}{1-q}:=P_{G / T}(q)$, where $\Phi^{+}$is the set of positive roots of $G$, and the $d_{i}$ are the invariant degrees of $G$. Further, $P_{G / T}(1)=\prod_{i} d_{i}=|W|$. Hence the hypothesis of (3.3) applies, and $G / T$ is mixed Tate, and has only even dimensional cohomology. By Lemma 3.1 (1), the variety $\mathcal{T}$ of maximal tori of $G$, which is the quotient of $G / T$ by the Weyl group $W$, is also mixed Tate.

Similar remarks apply to the spaces $G / P$ where $P$ is a parabolic subgroup of $G$. These spaces include Grassmann varieties and flag varieties. Moreover, the orbits $\{\mathcal{B}(w) \mid w \in W\}$ of $B$ on $\mathcal{B}$ are known to be affine spaces, and their closures $\overline{\mathcal{B}}(w)$ are the Schubert varieties. These form a collection of the type described in (3.1)(3), and are therefore mixed Tate (as is well known).

These examples can also be regarded as instances of the next result, which will also be used in $\S 5$.

Proposition (3.6). Let $p: X \longrightarrow Y$ be a smooth F-morphism of smooth varieties such that each fibre $p^{-1}(y)(y \in Y)$ is $F$-isomorphic to a fixed variety $Z$. Suppose that the local systems $R^{j} p_{*} \mathbb{C}$ induced by the map $X_{\sigma}(\mathbb{C}) \rightarrow Y_{\sigma}(\mathbb{C})$ are constant for each $j$. If any two of $X, Y, Z$ are mixed Tate, then so is the third.

Proof. We first remark that the condition on $R^{j} p_{*} \mathbb{C}$ implies that the $p$-adic étale local systems $R^{j} p_{*} \mathbb{Q}_{p}$ on $Y_{\bar{F}}$ are also constant. This follows from [SGA 4, XI Thm. 4.4]. It follows that the Leray spectral sequence has the form

$$
E_{2}^{i, j}=H^{i}\left(Y_{\bar{F}}, R^{j} p_{*} \mathbb{Q}_{p}\right) \stackrel{\sim}{\longrightarrow} H^{i}\left(Y_{\bar{F}}, \mathbb{Q}_{p}\right) \otimes_{\mathbb{Q}_{p}} H^{j}\left(Z_{\bar{F}}, \mathbb{Q}_{p}\right) \Rightarrow H^{i+j}\left(X, \mathbb{Q}_{p}\right) .
$$

It follows immmediately from this spectral sequence that if $Y$ and $Z$ are mixed Tate then so is $X$.

Suppose $X$ and $Z$ are mixed Tate. Let $v$ be a prime of $F$ and $q=q_{v}^{N_{v}}$ as in Proposition (3.3). We have to show that for almost all $v$, and $q$ sufficiently large, the eigenvalues of $\operatorname{Frob}_{v}^{N_{v}}$ on $H^{i}\left(Y_{\bar{F}}, \mathbb{Q}_{p}\right.$ ) (any $i$ ) are of the form $q^{m}$ for some integer $m$. For $i$ sufficiently large, $H^{i}\left(Y_{\bar{F}}, \mathbb{Q}_{p}\right)=0$, so there is nothing 
to prove. Thus by way of descending induction on $i$, we may assume that the assertion holds for $H^{n}\left(Y_{\bar{F}}, \mathbb{Q}_{p}\right)$ with $n>i$. Choose $j_{0}$ as large as possible so that $H^{j_{0}}\left(Z_{\bar{F}}, \mathbb{Q}_{p}\right) \neq 0$. For $r \geq 2$ the differential $d_{r}$ in the spectral sequence has the form $d_{r}: E_{r}^{i, j} \rightarrow E_{r}^{i+r, j-r+1}$. Suppose $M \subset H^{i}\left(Y_{\bar{F}}, \mathbb{Q}_{p}\right)$ is a Frob $v_{\text {-invariant }}$ submodule on which no eigenvalue of $\operatorname{Frob}_{v}^{N_{v}}$ is of the form $q^{m}$ for $N_{v}$ sufficiently large; then our induction hypothesis implies that $d_{2}\left(M \otimes_{\mathbb{Q}_{p}} H^{j_{0}}\left(Z_{\bar{F}}, \mathbb{Q}_{p}\right)\right)=0$. By the maximal property of $j_{0}$, no term of the form $E_{r}^{i, j_{0}}$ is the image of a differential in the spectral sequence. Hence by induction on $r \geq 2$, the same argument shows that for each $r$,

$$
M \otimes_{\mathbb{Q}_{p}} H^{j_{0}}\left(Z_{\bar{F}}, \mathbb{Q}_{p}\right) \subset E_{r+1}^{i, j_{0}}=\operatorname{ker}\left(d_{r}\right) \subset E_{r}^{i, j_{0}} .
$$

It follows that $M \otimes_{\mathbb{Q}_{p}} H^{j_{0}}\left(Z_{\bar{F}}, \mathbb{Q}_{p}\right)$ corresponds to a quotient of $H^{i+j_{0}}\left(X_{\bar{F}}, \mathbb{Q}_{p}\right)$. Since $X$ is mixed Tate, we conclude that $M=0$, so $Y$ is mixed Tate.

A similar argument shows that if $X$ and $Y$ are mixed Tate, then so is $Z$.

(3.7) Grothendieck-Springer fibres. In this section we regard $G$ as a reductive group scheme over the ring of integers in a number field $F$ (often $F$ can be taken to be $\mathbb{Q}$ ), and for any appropriate field $k$, we write $G(k)$ for the group of $k$-points of $G$. Let $x \in G(k)$; the Grothendieck-Springer fibre corresponding to $x$ is $\mathcal{B}_{x}^{G}:=\mathcal{B}_{x}=\{B \in \mathcal{B} \mid x \in B\}$. These varieties occur in many aspects of the representation theory of $G$ (see, e.g. $[\mathrm{Sp}]$ ) and related areas of the Langlands program such as the representation theory of the affine Hecke algebras (see, e.g. $[\mathrm{KLu}])$. The cohomology of the varieties $\mathcal{B}_{x}$ carries an action of the Weyl group $W$, which does not arise directly from an action of $W$ on $\mathcal{B}_{x}$; this is the celebrated "Springer action", and provides a vital link between $W$ and unipotent orbits in $G$. It is easy to reduce the study of the varieties to the case of unipotent $x$ using the Jordan decomposition. Let us therefore take $x$ to be unipotent. There is a description of the unipotent orbits in $G(k)$ which is largely independent of the field $k$. In particular, there is always a canonical injection from the unipotent orbits of $G(\mathbb{C})$ to those of $G(k)$ for any algebraically closed field $k$ (see, e.g. [Lu2]). Clearly the variety $\mathcal{B}_{x}$ depends on $x$ only up to conjugacy, so given a unipotent element $x \in G(\mathbb{C})$, it makes sense to speak of $x \in G(k)$. The variety $\mathcal{B}_{x}(k)$ for $G(k)$ may therefore be thought of as the $k$-points of an $F$-scheme $\mathcal{B}_{x}$.

We discuss briefly how these varieties fit into our picture. Fix a Borel subgroup $B=T U$, where $T$ is a maximal torus of $G$ and $U$ is the unipotent radical of $B$, and is a maximal unipotent subgroup of $G$. The element $x$ may be chosen in $U(k)$, where $k$ is the prime field. It is known from [Sp] (see also [Sh, Appendix A6]) that for all $i, H^{i}\left(\mathcal{B}_{x}, \mathbb{Q}_{p}\right) \cong H^{i}\left(\tilde{S}_{0}, \mathbb{Q}_{p}\right)$, where $S_{0}$ is the intersection of a Slodowy slice with the nilpotent cone in the Lie algebra of $G$, and $\tilde{S}_{0}$ is its preimage in the Grothendieck-Springer resolution. Since $\tilde{S}_{0}$ is smooth, it follows that in the notation of (3.3), 
(3.7.1). The eigenvalues of $\mathrm{Frob}_{v}$ on $H^{i}\left(\mathcal{B}_{x}, \mathbb{Q}_{p}\right)$ all have absolute value $q^{\frac{i}{2}}$.

Denote by $\ell$ the characteristic of the finite field $\mathbb{F}_{q}$. Consider the following statements.

(3.7.2). $\quad\left|\mathcal{B}_{x}\left(\mathbb{F}_{q^{m}}\right)\right|=P_{x}\left(q^{m}\right)$ for all $m$, for sufficiently large $\ell$ and $q$, where $P_{x}(t) \in \mathbb{Q}[t]$. Note that $\left|\mathcal{B}_{x}\left(\mathbb{F}_{q^{m}}\right)\right|=\operatorname{Ind}_{B\left(\mathbb{F}_{q^{m}}\right)}^{G\left(\mathbb{F}_{m}\right)}(1)(x)$.

(3.7.3). For almost all characteristics, $H^{i}\left(\mathcal{B}_{x}, \mathbb{Q}_{p}\right)=0$ for $i$ odd.

(3.7.4). $\mathcal{B}_{x}$ is mixed Tate.

(3.7.5). With $P_{x}(t)$ as in (3.7.2), $P_{x}(1)=\operatorname{dim} H^{*}\left(\mathcal{B}_{x}, \mathbb{Q}_{p}\right)$.

Note that in (3.7.2), $G\left(\mathbb{F}_{q^{m}}\right)$ is a finite group, and by the remarks above concerning the classification of unipotent orbits, $x$ may be interpreted as representing a unipotent class in $G\left(\mathbb{F}_{q^{m}}\right)$. The right side of $(3.7 .2)$ is the value of the indicated induced character of $G\left(\mathbb{F}_{q^{m}}\right)$ at $x$. All the above statements are known to be true, which shows that the varieties $\mathcal{B}_{x}$ are examples of the type in (3.3)(1) above. Our results show that all the statements follow from (3.7.2). This may be seen using the following elementary result.

Lemma (3.7.6). Fix an integer $d \geq 0$, and let $\xi_{1}, \ldots, \xi_{d}$ be complex numbers satisfying $\left|\xi_{i}\right|=1$ for each $i$, and for each positive integer $m, \sum_{i=1}^{d} \xi_{i}^{m}=c \in \mathbb{R}$, where $c$ is independent of $m$. If $c=0$ then $d=0$. If $c \neq 0$ then $c=d$ and $\xi_{i}=1$ for each $i$.

To see that (3.7.2) implies all the other properties of $\mathcal{B}_{x}$, let $\mathcal{F}$ be the relevant Frobenius morphism as in the proof of (3.3). The eigenvalues of $\mathcal{F}$ on $H^{i}\left(\mathcal{B}_{x}, \mathbb{Q}_{p}\right)$ may be written $\zeta_{j i} q^{\frac{i}{2}}, j=1, \ldots, d_{i}=\operatorname{dim} H^{i}\left(\mathcal{B}_{x}, \mathbb{Q}_{p}\right)$, where the $\zeta_{j i}$ have absolute value 1 . Write $d_{x}$ for the dimension of $\mathcal{B}_{x}$. Using the relationship between the cohomology of $\mathcal{B}_{x}$ and $\tilde{S}_{0}$, and Grothendieck's fixed point formula, we have

$$
\begin{aligned}
P_{x}\left(q^{m}\right)=\left|\mathcal{B}_{x}\left(\mathbb{F}_{q^{m}}\right)\right| & =\sum_{i}(-1)^{i} \operatorname{trace}\left(\mathcal{F}^{m}, H_{c}^{i}\left(\mathcal{B}_{x}, \mathbb{Q}_{p}\right)\right) \\
& =\sum_{i}(-1)^{i} \sum_{j=1}^{d_{i}} \zeta_{j i}^{m} q^{m \frac{2 d_{x}-i}{2}} \\
& =\sum_{i}(-1)^{i} q^{m\left(d_{x}-\frac{i}{2}\right)} \sum_{j=1}^{d_{i}} \zeta_{j i}^{m}
\end{aligned}
$$

If we write $P_{x}(t)=\sum_{k=0}^{d_{x}} c_{k} q^{d_{x}-k}$ (with $c_{k} \in \mathbb{Q}$ ), it follows that for $i$ odd, $\sum_{j=1}^{d_{i}} \zeta_{j i}^{m}=0$ for all $m$, and $\sum_{j=1}^{d_{2 k}} \zeta_{j, 2 k}^{m}=c_{k}$ for all $k$ and $m$. We deduce from 
(3.7.6) that if $i$ is odd, $H^{i}\left(\mathcal{B}_{x}, \mathbb{Q}_{p}\right)=0$. If $i=2 k$ is even, then (3.7.6) (with $c=c_{k}$ ) shows that if $d_{i} \neq 0, \zeta_{j i}=1$ for each $j$, and as in the proof of (3.3), it follows that $\mathcal{B}_{x}$ is mixed Tate, and (3.7.5) is clear.

The assertion (3.7.2) is known to be true essentially case by case, with the classical groups having been treated by Shoji and Srinivasan, while the exceptional groups have been treated by Shoji, Spaltenstein and Benyon. In [dLP], the authors prove (also using methods which are to some extent case by case) that $\mathcal{B}_{x}$ is stratified by affine spaces, which implies all of the above properties.

When $G=\mathrm{GL}_{n},(3.7 .2)$ goes back to Green [Gr], who deduced it using representation theory and combinatorics. It would be interesting to find a combinatorial approach to (3.7.2) in a more general situation. This would provide another proof that the cohomology of $\mathcal{B}_{x}$ vanishes in odd degrees and is spanned by Hodge classes.

We conclude our discussion of this example by noting that when $x$ is a regular element in a Levi subgroup $L$ of a parabolic subgroup of $G$, it follows from [Lu] that in $(3.7 .5), \operatorname{dim} H^{*}\left(\mathcal{B}_{x}\right)=|W / W(L)|$, where $W$ and $W(L)$ are respectively the Weyl groups of $G$ and $L$. In this case, it may be shown directly that $P_{x}(1)=$ $|W / W(L)|$. In general, the two sides of (3.7.5) are not known explicitly.

\section{$\S 4$ Minimally pure varieties}

Throughout this section, $X$ will be a variety of dimension $d$ over the number field $F$, and notation will be as in $\S \S 1$ and 2 . In particular, for each $j$ we have the weight filtration

$$
0 \subseteq W_{0} H_{c}^{j}\left(X, \mathbb{Q}_{p}\right) \subseteq W_{1} H_{c}^{j}\left(X, \mathbb{Q}_{p}\right) \subseteq \cdots \subseteq W_{2 d} H_{c}^{j}\left(X, \mathbb{Q}_{p}\right) .
$$

Definition (4.1) ((cf. $[D L],(5.1))$. Say that $X$ is minimally pure, (or $m p)$, if for each $j, \mathrm{Gr}_{2 j-2 d}^{W} H_{c}^{j}\left(X, \mathbb{Q}_{p}\right)=H_{c}^{j}\left(X, \mathbb{Q}_{p}\right)$.

Say that $X$ is Frobenius minimally pure (or fmp) if for any prime $v \notin S$, there is a power $\mathcal{F}=\operatorname{Frob}_{v}^{N}$ of Frob $_{v}$ such that the eigenvalues of $\mathcal{F}$ on $H_{c}^{j}\left(X, \mathbb{Q}_{p}\right)$ are all equal to $q_{v}^{N(j-d)}$.

Remark (4.1.1). It follows from Poincaré duality that when $X$ is smooth, then $X$ is $\mathrm{mp}$ if and only if $\mathrm{Gr}_{2 j}^{W} H^{j}\left(X, \mathbb{Q}_{p}\right)=H^{j}\left(X, \mathbb{Q}_{p}\right)$. In particular, it is rare for a proper smooth variety to be $\mathrm{mp}$.

The notion of mp variety was also introduced in [DL, §5] for varieties over $\overline{\mathbb{F}}_{q}$ in terms of eigenvalues of Frobenius. The results above may be used to show that the conditions mp and fmp are equivalent: 
Proposition (4.2). Let $X$ be as above. Then the following conditions are equivalent

(1) $X$ is fmp.

(2) $X$ is mixed Tate and $m p$.

(3) $X$ is $m p$ and $\operatorname{Gr}_{\mathbf{F}}^{m} \operatorname{Gr}_{\mathbf{F}}^{n} H_{c}^{j}\left(X_{\sigma}(\mathbb{C}), \mathbb{C}\right)=0$ unless $m=n=j-d .^{1}$

(4) $X$ is $m p$.

Proof. The equivalence (1) $\Leftrightarrow(2)$ follows from the fact that for any integer $w$, Frob $_{v}$ acts on $\mathrm{Gr}_{w}^{W} H_{c}^{j}\left(X, \mathbb{Q}_{p}\right)$ with eigenvalues of absolute value $q_{v}^{w}$.

That $(2) \Rightarrow(3)$ follows from Theorem (2.2)(2). Conversely, if (3) holds, then no weight can occur in the cohomology of $X$ in different degrees. In particular the eigenvalues of Frobenius in different cohomological degrees are disjoint. It now follows from Remark (2.4) that $X$ is mixed Tate, so (2) holds.

It is evident that $(3) \Rightarrow(4)$. The converse follows from the fact that for any $F$-scheme $Y$ of dimension $d, \operatorname{Gr}_{\mathbf{F}}^{r} \operatorname{Gr}_{\mathbf{F}}^{s} H_{c}^{j}\left(Y_{\sigma}(\mathbb{C}), \mathbb{C}\right)=0$ unless $r, s \geq j-d$. To see this, note that by Nagata's theorem we may embed $Y$ as a dense open subscheme of a proper $F$-scheme $\bar{Y}$. Hence, by excision, it suffices to consider the case $Y$ proper, and for this case the result follows from [De 2, Thm. 8.2.4(ii)].

Examples (4.3). There are many examples of minimally pure (and hence fmp) varieties in [DL]. We mention some below, and use the results of $\S 3$ above to point out that some other well known varieties associated with algebraic groups are mixed Tate .

(1) Among the $\mathrm{mp}$ varieties in [DL] are: hyperplane complements, complements in algebraic tori of unions of kernels of characters (toral complements), toric varieties, $G / T$, where $T$ is a maximal torus of the reductive group $G$, and varieties constructed from these.

(2) Let $G$ be a complex connected reductive algebraic group, and let $\mathfrak{G}$ be its Lie algebra. Let $G_{r s}, \mathfrak{G}_{r s}$ respectively denote their varieties of regular semisimple elements (see [LS]). Then both these varieties are mixed Tate in the above sense. To see this, note that (see, e.g. [LS, §2]) if $T$ is a maximal torus of $G$, then

$$
G_{r s} \cong G / T \times{ }_{W} T_{r s}=\left(G / T \times T_{r s}\right) / W,
$$

where $T_{r s}$ is the variety of regular elements of $T$, which is the complement of the kernels of the roots in $T$. We have seen that $G / T$ and $T_{r s}$ are mixed Tate (in fact $T_{r s}$ is $\mathrm{mp}$ - see below), and hence by (3.1), so is $G_{r s}$. A similar argument applies to $\mathfrak{G}_{r s}$.

\footnotetext{
${ }^{1}$ The condition $\operatorname{Gr}_{\mathbf{F}}^{m} \operatorname{Gr}_{\mathbf{F}}^{n} H_{c}^{j}=0$ if $m \neq n$ is called Tate by some authors. The discussion of $\S 2.4$ shows that it is conjecturally equivalent to our condition mixed Tate
} 
Corollary (4.4). If $X$ is minimally pure then $X$ satisfies the conditions of (3.3)(2) with $\varepsilon(X)=(-1)^{\operatorname{dim} X}$.

Proof. By Proposition 4.2, $X$ is fmp, and hence by Grothendieck's fixed point theorem, for almost all sufficiently large $q$ and all $m$,

$$
\left|X\left(\mathbb{F}_{q^{m}}\right)\right|=\sum_{j}(-1)^{j} \operatorname{trace}\left(\operatorname{Frob}_{q}, H_{c}^{j}\left(X, \mathbb{Q}_{p}\right)=\sum_{j}(-1)^{j} \operatorname{dim} H_{c}^{j}\left(X, \mathbb{Q}_{p}\right) q^{m(j-d)},\right.
$$

where $d=\operatorname{dim} X$. Thus $\left|X\left(\mathbb{F}_{q^{m}}\right)\right|=P\left(q^{m}\right)$ where $P(t) \in \mathbb{Q}[t]$. and $P(-1)=$ $(-1)^{d} \sum_{j} \operatorname{dim} H_{c}^{j}\left(X, \mathbb{Q}_{p}\right)$, as asserted.

Proposition (4.5). Let $p: X \longrightarrow Y$ satisfy the conditions of Proposition (3.6). If $X$ and $Z$ (resp. $Y$ and $Z$ ) are $m p$ then so is $Y$ (resp. $X$ ). In either of these cases the Leray spectral sequence for $p$ degenerates at $E_{2}$.

Proof. This follows from an analysis of the spectral sequence, analogous to that in the proof of (3.6). If $Y$ and $Z$ are mp then one sees easily that $X$ is mp.

If $X$ and $Z$ are mp, then one proves by descending induction on $i$ that $H^{i}\left(Y_{\bar{F}}, \mathbb{Q}_{p}\right)$ is pure of weight $2 i$. To see this consider the differential $d_{r}: E_{r}^{i, j} \rightarrow E_{2}^{i+r, j-r+1}$. If $H^{i+r}\left(Y_{\bar{F}}, \mathbb{Q}_{p}\right)$ is pure of weight $2(i+r)$ then $E_{2}^{i+r, j-r+1}$ is pure of weight $2(i+j+1)$ because $Z$ is $\mathrm{mp}$, while all weights appearing in $E_{r}^{i, j}$ are at most $2(i+j)$. Hence $d_{r}=0$, the spectral sequence degenerates at $E_{2}$, and the proposition follows easily.

\section{$\S 5$ Real points of COMPLEX VARIETiEs}

In this section, $X$ remains a variety defined over a number field $F$ as above, but we assume further that $X$ has a real structure, i.e. that there is an embedding $F \subset \mathbb{R}$. In this case $X(\mathbb{C})$ has a real structure, defined by complex conjugation $\sigma: X(\mathbb{C}) \longrightarrow X(\mathbb{C})$. The space $X(\mathbb{C})^{\sigma}$ of fixed points of $\sigma$ on $X(\mathbb{C})$ will be referred to as the space $X(\mathbb{R})$ of real points of $X$. It is well known by a theorem of Whitney that $X(\mathbb{R})$ is a finite union of its connected components. The study of the number and nature of these components is an active branch of real algebraic geometry.

In this section we point out some connections between the real case and the points of $X$ over a finite field.

Proposition (5.1). Suppose $X$ is as in the first paragraph of this section and assume that $X$ is mixed Tate. Let $\eta(X)= \pm 1$ and assume that for $m, j \geq 0 \sigma$ acts on $\operatorname{Gr}_{\mathbf{F}}^{m} \operatorname{Gr}_{\overline{\mathbf{F}}}^{m} H_{c}^{j}(X, \mathbb{C})$ by $\eta(X)(-1)^{m}$.

If $P(q)$ is the polynomial counting $X\left(\mathbb{F}_{q}\right)$ for sufficiently large $q$ (cf. (3.4)), then $P(-1)=\eta(X) \chi_{c}(\mathbb{R})$ where $\chi_{c}$ denotes the Euler characteristic with compact 
support. In particular, if $\chi_{c}(X(\mathbb{R}))= \pm \operatorname{dim} H_{c}^{*}(X, \mathbb{C})$ then

$$
P(-1)=\eta(X) \chi_{c}(X(\mathbb{R}))= \pm \operatorname{dim} H_{c}^{*}(X, \mathbb{C})
$$

and $X$ satisfies the conditions of (3.3)(2).

Proof. In general $\sigma$ acts on $H_{c}^{j}(X, \mathbb{C})$, transforming the Hodge filtration into its dual. It therefore induces isomorphisms (see $[\mathrm{H}, \S 15, \mathrm{p} .120]$ )

$$
\operatorname{Gr}_{\mathbf{F}}^{m} \operatorname{Gr}_{\overline{\mathbf{F}}}^{n} H_{c}^{j}(X, \mathbb{C}) \longrightarrow \operatorname{Gr}_{\mathbf{F}}^{n} \operatorname{Gr}_{\overline{\mathbf{F}}}^{m} H_{c}^{j}(X, \mathbb{C}),
$$

and in particular acts on $\operatorname{Gr}_{\mathbf{F}}^{m} \operatorname{Gr}_{\overline{\mathbf{F}}}^{m} H_{c}^{j}(X, \mathbb{C}$ ) with eigenvalues \pm 1 (see, e.g. $[\mathrm{H}$, $\S 15]$, or [DL, $\S 1])$.

Hence, our hypothesis gives

$$
\begin{aligned}
\chi_{c}(X(\mathbb{R})) & =\sum_{m, j}(-1)^{j} \operatorname{Trace}\left(\sigma, \operatorname{Gr}_{\mathbf{F}}^{m} \operatorname{Gr}_{\overline{\mathbf{F}}}^{m} H_{c}^{j}(X, \mathbb{C})\right) \\
& =\eta(X) \sum_{m, j}(-1)^{j+m} \operatorname{dim}\left(\operatorname{Gr}_{\mathbf{F}}^{m} \operatorname{Gr}_{\overline{\mathbf{F}}}^{m} H_{c}^{j}(X, \mathbb{C})\right) .
\end{aligned}
$$

Further, by the Grothendieck fixed point theorem,

$$
P(q)=\sum_{m, j}(-1)^{j} \operatorname{dim}\left(\operatorname{Gr}_{\mathbf{F}}^{m} \operatorname{Gr}_{\overline{\mathbf{F}}}^{m} H_{c}^{j}(X, \mathbb{C})\right) q^{m},
$$

whence $P(-1)=\sum_{m, j}(-1)^{j+m} \operatorname{dim}\left(\operatorname{Gr}_{\mathbf{F}}^{m} \operatorname{Gr}_{\overline{\mathbf{F}}}^{m} H_{c}^{j}\left(X, \mathbb{Q}_{p}\right)\right)=\eta(X) \chi_{c}(X(\mathbb{R}))$. This proves the proposition.

We say that a mixed Tate variety $X$ over a real number field is computable if $P(-1)=\chi_{c}(X(\mathbb{R})) ; X$ will be called strongly computable of index $\varepsilon(X)= \pm 1$ if $P(-1)=\chi_{c}(X(\mathbb{R}))=\varepsilon(X) \operatorname{dim} H_{c}^{*}(X, \mathbb{C})$. From (4.4) it follows that any variety $X$ which is computable and mp is strongly computable, with index $(-1)^{\operatorname{dim} X}$.

Note that the Lefschetz fixed point theorem [DL, §1.4] implies that

$$
\chi_{c}(X(\mathbb{R}))=\sum_{j}(-1)^{j} \operatorname{Trace}\left(\sigma, H_{c}^{j}(X, \mathbb{C})\right) .
$$

Hence the condition $\chi_{c}(X(\mathbb{R}))= \pm \operatorname{dim} H_{c}^{*}(X, \mathbb{C})$ is equivalent to asking that for each $j, \sigma$ acts on $H_{c}^{j}(X, \mathbb{C})$ by $\eta^{\prime}(X)(-1)^{j}$ with $\eta^{\prime}(X)= \pm 1$.

Our next result gives some sufficient conditions for computability.

Proposition (5.2). Let $F \subset \mathbb{R}$ be a number field.

(1) Affine space $\mathbb{A}^{n}$ is strongly computable of index $(-1)^{n}$.

(2) If $X$ and $Y$ are (strongly) computable, so is $X \times Y$. 
(3) Suppose $L:=\left(X_{\lambda}\right)_{\lambda \in \Lambda}$ is an Eulerian collection (cf. [DL, (2.1)]) of closed subvarieties of $X$, all defined over the number field $F \subseteq \mathbb{R}$. If all the varieties $X_{\lambda}$ are strongly computable, then so is $X^{0}:=X \backslash \cup_{Y \in L, Y \neq X} Y$.

(4) Complex hyperplane complements defined over $F$ are strongly computable.

(5) Algebraic tori which are defined and split over $F$, are strongly computable.

(6) Toric varieties defined over $F$ are strongly computable.

(7) Linear algebraic groups over $F$ whose reductive quotient is split over $F$ are computable.

Proof. (1) is evident, and (2) follows from the Künneth theorem. For (3), recall that $L$ is a collection of closed subvarieties $\left(X_{\lambda}\right)$ of $X$, which is closed under intersection. It is known [DL, Cor. 2.3] that if $\chi$ is any additive function on the Boolean algebra $\mathcal{A}$ of subvarieties of $X$ which is generated by $\left\{X_{\lambda}\right\}$, i.e. $\chi$ satisfies $\chi(Z)+\chi(Y \backslash Z)=\chi(Y)$ for $Y, Z \in \mathcal{A}$, then for any $Y \in L$, if $Y^{0}:=Y \backslash \cup_{Z \in \mathcal{A}, Z \subset Y, Z \neq Y} Z$, then $\chi\left(Y^{0}\right)=\sum_{Z \in L, Z \subset Y} \mu(Z, Y) \chi(Z)$, where $\mu$ is the (combinatorially defined) Möbius function of $L$. Since both $\chi(Y)=\chi_{c}(Y(\mathbb{R})$ ) and $\chi^{\prime}(Y)=P_{Y}(-1)$ are additive, it follows that if they are equal on all $X_{\lambda}$, which is our hypothesis, then they are equal on all $Y^{0}$, and in particular on $X^{0}$. This proves (3).

The statements (4),(5) and (6) are immediate consequences of (1),(2) and (3), as well as known facts about the action of $\sigma$ on the cohomology of hyperplane complements. Note that a one-dimensional torus is a hyperplane complement, and hence any connected split torus is a product of hyperplane complements.

Finally, any linear algebraic group $G$ is the product $G=G_{R} R_{u}(G)$ of a reductive group $G_{R}$ and the unipotent radical $R_{u}(G)$. Since $R_{u}(G)$ is an affine space, we are reduced by (1) and (2) to the case where $G$ is reductive. Now any reductive algebraic group $G$ is the disjoint union of its Bruhat cells $B w B$, where $B$ is a Borel subgroup and $w$ runs over the elements of the Weyl group. But $B w B$ is the product of an affine space and a split torus, whence it is computable. Hence $G$ is computable. This case may also be seen as an instance of (3).

We illustrate the above with the following (known) result.

Corollary (5.3). ([Z], [OS]) Let $M$ be the complement of a complex hyperplane arrangement which is defined over a real algebraic number field. The number of components of $M(\mathbb{R})$ is the dimension of the cohomology ring of $M$. It is also equal to $\pm P(-1)$, where $P(q)$ is the (generic) number of points of $M$ over $\mathbb{F}_{q}$.

Proof. In the case of (5.3), the connected components of $M(\mathbb{R})$ are convex subsets of $\mathbb{R}^{n}$, and hence are homeomorphic to $\mathbb{R}^{n}$. Thus for each connected component $C$, we have $\chi_{c}(C)=(-1)^{n}=(-1)^{\operatorname{dim} M}=\varepsilon(M)$. The result is now a simple consequence of $(5.2)(4)$. 
Of course we have results similar to (5.3) for any variety constructed from those in $(5.2)$, but in general the connected components of $X(\mathbb{R})$ may differ from each other even when $X$ is smooth. In particular, all the statements of (5.3) remain valid when $M$ is the complement in a split torus of the union of the kernels of a finite set of characters defined over $F$. The statement analogous to (5.3) for this case is, we believe, new.

To deal with homogeneous spaces we have the following result.

Proposition (5.4). (cf. [DL, Thm. 6.1]) Let $F$ be real, and $p: X \rightarrow Y a$ morphism of F-varieties satisfying the conditions of Proposition (3.6). If any two of $X, Y, Z$ are computable then so is the third.

Proof. Write $P_{X}(q)=\left|X\left(\mathbb{F}_{q}\right)\right|$ etc. We saw in the proof of Proposition (3.6) that the terms of the Leray spectral sequence for $p$ are tensor products of the cohomology of $Y$ and $Z$. Hence $P_{X}(q)=P_{Y}(q) P_{Z}(q)$.

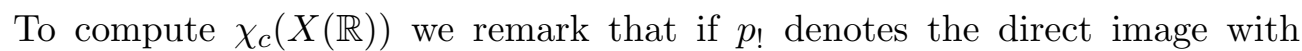
compact supports under $p$, then the conditions of (3.6) imply that for $j \geq 0$, $R^{j} p ! \mathbb{C}$ is a local system of $\mathbb{C}$-vector spaces on $Y$, which is dual to the local system $R^{2 d-j} p_{*} \mathbb{C}$, where $d=\operatorname{dim} X$, and hence constant. It follows that the terms of the Leray spectral sequence for compactly supported Betti cohomology have the form

$$
E_{2}^{i, j}=H_{c}^{i}\left(Y_{\sigma}(\mathbb{C}), R^{j} p_{!} \mathbb{C}\right) \stackrel{\sim}{\longrightarrow} H_{c}^{i}\left(Y_{\sigma}(\mathbb{C}), \mathbb{C}\right) \otimes_{\mathbb{C}} H_{c}^{j}\left(Z_{\sigma}(\mathbb{C}), \mathbb{C}\right) .
$$

Writing $\chi_{c}(X(\mathbb{R}))$ as the alternating sum of traces of complex conjugation on compactly supported Betti cohomology, as in the proof of Proposition (5.1), we find that $\chi_{c}(X(\mathbb{R}))=\chi_{c}(Y(\mathbb{R})) \chi_{c}(Z(\mathbb{R}))$.

The proposition now follows, since by (3.6) if two of $X, Y, Z$ are mixed Tate then so is the third.

Corollary (5.5). Let $G$ be a connected (i.e. irreducible) linear algebraic group over the real number field $F$ and suppose $H$ is a closed $F$-subgroup. Suppose that both $G$ and $H$ have $F$-split reductive quotients. Then the homogeneous space $G / H$ is computable.

Proof. If $H$ is connected, the canonical projection $p: G \longrightarrow G / H$ satisfies the assumption (5.4). It follows from (5.2)(7) that $G / H$ is computable. In general, if $H^{0}$ is the connected component of $1 \in H$, then writing $W$ for the finite group $H / H^{0}$, we have $H_{c}^{*}(G / H) \cong H_{c}^{*}\left(G / H^{0}\right)^{W}$ for all cohomology theories we have considered. The statement now follows from the connected case.

The result (5.5) shows in particular that Grassmannian varieties are computable, and satisfy the conditions of $(3.3)(1)$. Note that these examples are not strongly computable, since in particular, when $H$ above is parabolic, $G / H$ has only even cohomology, and $P_{G / H}(1)=\operatorname{dim}_{c}^{*}(G / H) \neq P_{G / H}(-1)$. A simple example is when $G / H=\mathbb{P}^{n}$. 
Example (5.6). Let $X$ be the variety of maximal tori of $\mathrm{SL}_{2}$; we have seen above that $X$ is fmp, and $X$ is obviously smooth. This is an example of the type in (5.5), and it is easy to see (cf. [LvH, §10]) that $X \cong\left\{[x, y, z] \in \mathbb{P}^{2}\right.$ $\left.x^{2}+y z \neq 0\right\}$. We therefore have $P(q)=\left|X\left(\mathbb{F}_{q}\right)\right|=q^{2}$, and according to Corollary $(5.5)$, we have $\chi_{c}(X(\mathbb{R}))=1$. In this case one checks easily that $X(\mathbb{R})$ has two connected components $C_{1} \simeq \mathbb{P}^{1}(\mathbb{R}) \times \mathbb{R}$ and $C_{2} \simeq \mathbb{R}^{2}$, and that $\chi_{c}\left(C_{1}\right)=0$, while $\chi_{c}\left(C_{2}\right)=1$, confirming (5.5). A similar argument shows that for any connected reductive group $G$, its variety $X$ of maximal tori is strongly computable, and $\chi_{c}(X(\mathbb{R}))=(-1)^{N}$, where $N$ is the number of positive roots of $G$.

We next discuss a result relating $X(\mathbb{C})$ to $X\left(\mathbb{F}_{q}\right)$ in case $X$ is smooth and complete, and defined over a number field $F$. In this case $X(\mathbb{C})$ is compact in the analytic topology. Recall $([\mathrm{H}, \S 8.2])$ that if $M^{4 k}$ is a compact oriented $4 k$ dimensional manifold, there is a symmetric bilinear form on $H^{2 k}\left(M^{4 k}, \mathbb{R}\right)$ whose signature $\tau\left(M^{4 k}\right)$ is an invariant of $M^{4 k}$, called its index. When $X$ is as complete and even (say $2 k$-)dimensional, then $X(\mathbb{C})$ may be considered as a real $4 k$-dimensional manifold as above, and we may therefore speak of its index $\tau(X)$.

Proposition (5.7). Let $X$ be a smooth and complete mixed Tate variety over a number field $F$, and assume that $X(\mathbb{C})$ is a Kähler manifold (for example $X$ projective). Let $P(t) \in \mathbb{Q}[t]$ be such that for most $q, P(q)=\left|X\left(\mathbb{F}_{q}\right)\right|$ (see (3.3)). Then the index $\tau(X)=P(-1)$.

Proof. It follows from [H, Theorem 15.8.2] that if

$$
h^{m, n}=\operatorname{dim} \operatorname{Gr}_{\mathbf{F}}^{m} \operatorname{Gr}_{\mathbf{F}}^{n} H^{*}(X(\mathbb{C}), \mathbb{C}),
$$

then $\tau(X)=\sum_{m, n}(-1)^{n} h^{m, n}$. But with our assumptions, $h^{m, n}=0$ unless $m=n$, and $P(q)=\sum_{n} h^{n, n} q^{n}$. The result is now clear.

\section{REFERENCES}

[dLP] C. De Concini, G Lusztig and C. Procesi, Homology of the zero-set of a nilpotent vector field on a flag manifold., J. Amer. Math. Soc.1 (1988), 15-34.

[De] P. Deligne, Theorie de Hodge II, Inst. des Hautes Études Sci. Publ. Math. 40 (1971), 5-58.

[De 2] P. Deligne, Theorie de Hodge III. ,Inst. des Hautes Études Sci. Publ. Math. 44 (1974), 5-77.

[De 3] P. Deligne, Poids dans la cohomologie des variétés algebriques, Proceedings of the International Congress of Mathematicians (Vancouver 1974) 1 (1975),pp.79-85.

[Di] A. Dimca, Singularities and topology of hypersurfaces., Universitext. Springer-Verlag, New York, 1992.

[DL] A. Dimca and G.I. Lehrer, Purity and equivariant weight polynomials, Algebraic groups and Lie groups, Austral. Math. Soc. Lect. Ser., Cambridge Univ. Press, Cambridge 9 (1997), pp. $161-181$.

[EvdB] S. Edixhoven, T. van den Bogaart, Algebraic stacks whose number of points over finite fields is a polynomial, Number fields and function fields-Two parallel worlds (Texel 2004), Progress in Math. 239, 2005. 
[Fa] G. Faltings, Almost étale extensions, Cohomologies p-adiques et applications arithmétiques II, Astérisque 279 (2002), 185-270.

[Fo1] J-M. Fontaine, Le corps des périodes p-adiques, Périodes p-adiques, Astérisque 223, Société Mathématique de France, pp. 59-111, 1994.

[Fo2] J-M. Fontaine, Représentations p-adiques semi-stables, Périodes p-adiques, Astérisque 223, Société Mathématique de France, pp. 113-184, 1994.

[Gr] J.A. Green, The characters of the finite general linear groups, Trans. Amer. Math. Soc 80 (1955), 402-447.

[H] F. Hirzebruch, Topological methods in algebraic geometry. Grundlehren der math. Wiss., Third Edition., Springer-Verlag, Berlin Heidelberg New York, 1978.

[KLu] D. Kazhdan and G. Lusztig, Proof of the Deligne-Langlands conjecture for Hecke algebras., Invent. Math. 87 (1987), 153-215.

[Ki] M. Kisin, Potentially semi-stability of p-adic étale cohomology, Israel J. Math. 129 (2002), 157-174.

[KL] M. Kisin, G.I. Lehrer, Equivariant Poincaré polynomials and counting points over finite fields., J. Algebra 247 (2002), 435-451.

[KW] M. Kisin, S. Wortmann, A note on Artin motives, Math. Res. Letters 10 (2003), 375-389

[La] Serge Lang, Algebraic number theory. Second edition. Graduate Texts in Mathematics, 110., Springer-Verlag, New York, 1994.

[L] G.I. Lehrer, Rational tori, semisimple orbits and the topology of hyperplane complements, Comment. Math. Helv. 67 (1992), 226-251.

[LS] G.I. Lehrer and G.B. Segal, Homology stability for classical regular semisimple varieties, Math. Z. 236 (2001), 251-290.

[LvH] G.I. Lehrer and J. van Hamel, Euler characteristics of the real points of certain varieties of algebraic tori (2005), (to appear).

[Lu] G. Lusztig, An induction theorem for Springer's representations, Representation theory of Algebraic Groups and Quantum Groups, Adv. Stud. Pure Math. 40, Math. Soc. Japan, Kinokuniya (2004), 253-259.

[Lu2] G. Lusztig, Unipotent elements in small characteristic (2005), (to appear).

[OS] P. Orlik and L. Solomon, Combinatorics and topology of complements of hyperplanes, Invent. Math. 56 (1980), 167-189.

[SGA 4] M. Artin, A. Grothendieck and J.L Verdier Théorie des topos et cohomologie étale des schémas III, Lecture notes in Mathematics 305, Springer, 1973.

[Se] S. Sen, Continuous cohomology and p-adic Galois representations, Invent. Math. 62 (1980), 89-116.

[Sh] T. Shoji, On the Green polynomials of classical groups, Invent. Math. 74 (1983), 239-267.

[Sp] T.A. Springer, Trigonometric sums, Green functions of finite groups and representations of Weyl groups., Invent. Math. 36 (1976), 173-207.

[Z] T. Zaslavsky, Facing up to arrangements: face-count formulas for partitions of space by hyperplanes, Mem. Amer. Math. Soc. no. 154, Amer. Math. Soc., 1975.

Mark Kisin

Department of Mathematics, University of Chicago.

E-mail: kisin@math.uchicago.edu

Gus Lehrer

School of Mathematics and Statistics, University of Sydney.

E-mail: G.Lehrer@maths.usyd.edu.au 